Jean-Pierre Wolf is at the Group of Applied Physics, University of Geneva, 22 Chemin de Pinchat, 1211 Geneve 4, Switzerland. Yaron Silberberg is in the Department of Physics and Complex Systems, Weizmann Institute of Science, Rehovot 76100, Israel.

e-mail: Jean-Pierre.Wolf@unige.ch; yaron.silberberg@weizmann.ac.il
References

1. Silberberg, Y. Annu. Rev. Phys. Chem. 60, 277-292 (2009).

2. Roth, M. et al. Phys. Rev. Lett. 102, 253001 (2009).

3. Bonacina, L., Trisorio, A., Hauri, C. \& Wolf, J.-P. Phys. Chem. Chem. Phys. 14, 9317-9322 (2012).

4. Afonina, S. et al. Appl. Phys. B 111, 541-549 (2013).

5. Kalashnikov, D. A., Paterova, A. V., Kulik, S. P. \& Krivitsky, L. A. Nature Photon. 10, 98-101 (2016).

6. Barreto Lemos, G. et al. Nature 512, 409-412 (2014)

7. Mandel, L. Opt. Lett. 16, 1882-1883 (1991).
8. Zou, X. Y., Wang, L. J. \& Mandel, L. Phys. Rev. Lett. 67, 318-321 (1991)

9. Elitzur, A. C. \& Vaidman, L. Foundations Phys. 23, 987-997 (1993).

10. Herbst, T. et al. Proc. Natl Acad. Sci. USA 112, 14202-14205 (2015).

11. Dayan, B., Pe’er, A., Friesem, A. \& Silberberg, Y. Phys. Rev. Lett. 94, 043602 (2005).

12. Shapiro, J. H., Venkatraman, D. \& Wong, F. N. C. Sci. Rep. 5, 10329 (2015)

\title{
INFRARED OPTICS
}

\section{Nanoscale heat}

Radiative heat transfer via the emission of light, which largely involves photons at infrared wavelengths, is a well-known phenomenon taught in undergraduate physics courses. Yet, the effect is still not fully understood when it occurs on the nanoscale. Studies have shown that the rate of thermal radiation by light can be modified by altering the optical properties of structures and can dramatically deviate away from the values expected from conventional black-body physics. In particular, enhanced heat transfer has been achieved via structures such as diffraction gratings and small gaps between surfaces supporting modes at infrared wavelengths. Unfortunately, it is difficult to quantify the effects for gaps smaller than a few tens of nanometres. And, not all of the theoretical studies on nanoscale heat transfer are in agreement. Kyeongtae Kim, Bai Song, Víctor Fernández-Hurtado and colleagues from the USA, Spain and South Korea have now experimentally demonstrated a technique to measure radiative heat transfer for gaps as small as $2 \mathrm{~nm}$ using custom probes with embedded thermocouples (Nature 528, 387-391; 2015).

One of the authors of the study, Pramod Reddy, told Nature Photonics that the motivation of this work was to elucidate the principles governing the exchange of thermal radiation between objects separated by gaps as small as a few nanometres and to establish the basic laws that can be applied to a great variety of nanoscale systems and devices. Reddy explained that one of the most important findings is the unambiguous demonstration that the theory of fluctuational electrodynamics provides an accurate description of the radiative heat transfer all

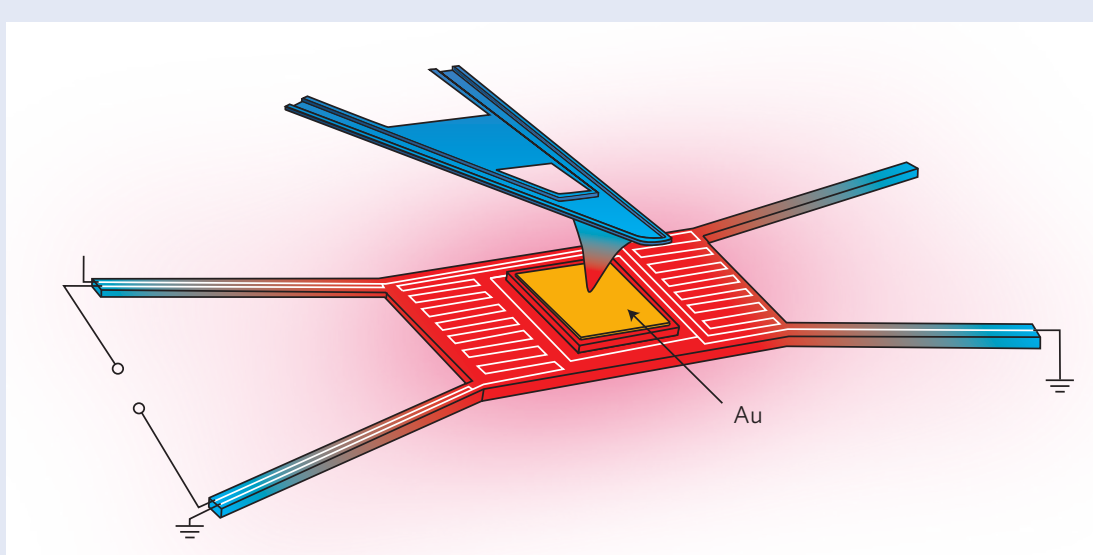

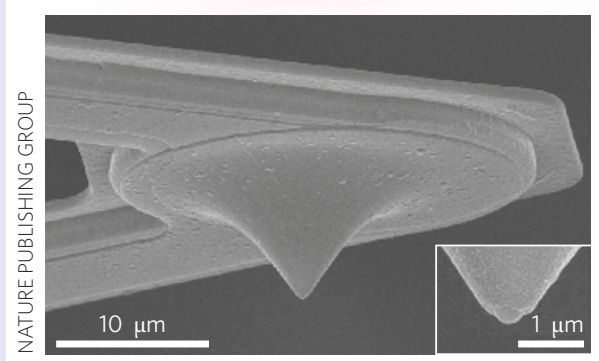

the way down to separations of a few nanometres.

According to Reddy, it was a real challenge to measure the very small heat flux between the gold surfaces in their experiment, which was beyond the sensitivity of existent techniques. "This required the combination of our scanning probes with microdevices capable of periodic temperature modulations, which led to an increase in resolution by more than an order of magnitude. This improvement was crucial to establish a definitive picture of the radiative heat transfer between metals at the nanoscale," Reddy explained.

"Our results demonstrated that in nanometre-scale gaps the radiative heat

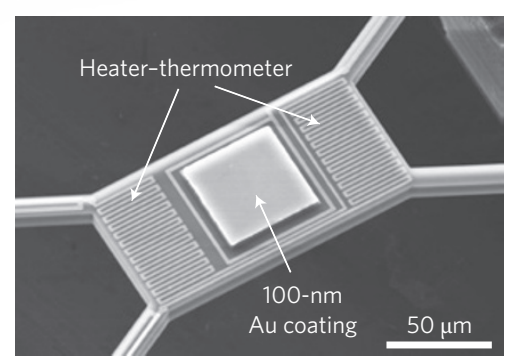

fluxes can be many orders of magnitude larger than what it is expected from the Stefan-Boltzmann law for black bodies," Reddy told Nature Photonics. "This discovery can be extremely useful for technologies that make use of thermal radiation such as thermophotovoltaics, heat-assisted magnetic recording or nanolithography."

The team now hopes to use their findings to tackle a variety of open problems such as the active control of thermal radiation in nanoscale devices and applying nanostructuring to shape the spectral characteristics of thermal emissions.

DAVID PILE 